\title{
Endoscopic ultrasound-guided transvascular needle biopsy of thoracic and abdominal lesions: a multicenter experience
}

\section{(ㄷ)(i) $\Theta$}

Authors

Albert Garcia-Sumalla ${ }^{1}$, Jose C. Subtil ${ }^{2}$, Carlos de la Serna ${ }^{3}$, Sandra Maisterra ${ }^{1}$, Jose Ramon Aparicio ${ }^{4}$, Alejandro Enrique Bojorquez ${ }^{2}$, Rafael Leon Montañes ${ }^{5}$, Enrique Vazquez-Sequeiros ${ }^{6}$, Joan B. Gornals ${ }^{1,7}$

Institutions

1 Endoscopy Unit, Department of Digestive Diseases, Hospital Universitari de Bellvitge, Bellvitge Biomedical Research Institute (IDIBELL), University of Barcelona, Barcelona, Catalonia, Spain

2 Endoscopy Unit, Department of Digestive Diseases, Complejo Universitario de Navarra, Pamplona, Spain

3 Endoscopy Unit, Department of Digestive Diseases, Hospital Universitario Rio Hortega, Valladolid, Spain

4 Endoscopy Unit, Department of Digestive Diseases, Hospital General Universitario de Alicante, Alicante, Spain

5 Endoscopy Unit, Department of Digestive Disease, Hospital Universitario Virgen del Rocio, Sevilla, Spain

6 Endoscopy Unit, Department of Digestive Diseases, Hospital Universitario Ramon y Cajal, Madrid, Spain

7 Health Science Department, Universitat Oberta de Catalunya, Barcelona, Catalonia, Spain

submitted 8.7.2020

accepted after revision $\quad 21.9 .2020$

\section{Bibliography}

Endoscopy International Open 2020; 08: E1900-E1908

DOI 10.1055/a-1288-0030

ISSN 2364-3722

(C) 2020. The Author(s).

This is an open access article published by Thieme under the terms of the Creative Commons Attribution-NonDerivative-NonCommercial License, permitting copying and reproduction so long as the original work is given appropriate credit. Contents may not be used for commecial purposes, or adapted, remixed, transformed or built upon. (https://creativecommons.org/licenses/by-nc-nd/4.0/)

\section{Corresponding author}

Joan B. Gornals, MD, PhD, Endoscopy Unit, Department of Digestive Diseases, Hospital Universitari de Bellvitge-IDIBELL (Bellvitge Biomedical Research Institute), Feixa Llarga s/n, 08907 L'Hospitalet de Llobregat, Barcelona, Catalonia, Spain Fax: +34932607681

jgornals@bellvitgehospital.cat $\bigoplus$ Supplementary material is available under https://doi.org/10.1055/a-1288-0030

\section{ABSTRACT}

Background and study aims Traditionally in the case of a vascular interposition, endoscopic ultrasound-guided fineneedle aspiration (EUS-FNA) has been contraindicated. A transvascular route (TV) is feasible and probably a safe alternative approach in selected patients, but data are scarce. The primary aim of this study was to analyze the diagnostic yield and safety of EUS-TV-FNA in thoracic and abdominal lesions. Secondary aims included evaluation of the clinical impact and technical aspects.

Patients and methods A retrospective multicenter study was conducted with inclusion of all consecutive patients that underwent EUS-TV-FNA from July 2007 to January 2020. Feasibility, cytopathology, procedure details, and safety were evaluated. Univariate analysis was performed to identify variables associated with incidents, cytopathological diagnosis, and clinical impact.

Results Data were collected from a total of 49 cases and 50 EUS-TV-FNAs. The aorta $(n=19)$ and portal system $(n=17)$ were the most frequently punctured. The most frequent lesions were mediastinal lymph nodes $(n=13)$ and pancreatic tumors $(n=11)$. The diagnostic yield was $86 \%$, and there were nondiagnostic samples in seven cases. Overall sensitivity, specificity, and accuracy were $88 \%(95 \% \mathrm{Cl}, 0.74-$ 0.96), $100 \%(95 \% \mathrm{Cl}, 0.59-1)$, and $90 \%(95 \% \mathrm{Cl}, 0.78-0.96)$, respectively. Only three incidents were detected: two mural hematomas and a self-limited bleeding of gastroduodenal artery. In most patients, there was a significant impact on clinical management (88\%). Arterial vessel and ASA-III had a trend with incidents (both, $P<0.08$ ). Rapid on-site evlauation was found to be an independent predictor for obtaining a conclusive sample (OR 6.2; $95 \% \mathrm{Cl}, 1.06-36.73, P<$ 0.04).

Conclusions EUS-TV-FNA is feasible, seems to be safe, and can be recommended when no other targets are available, and the information obtained would impact on the clinical plan. 

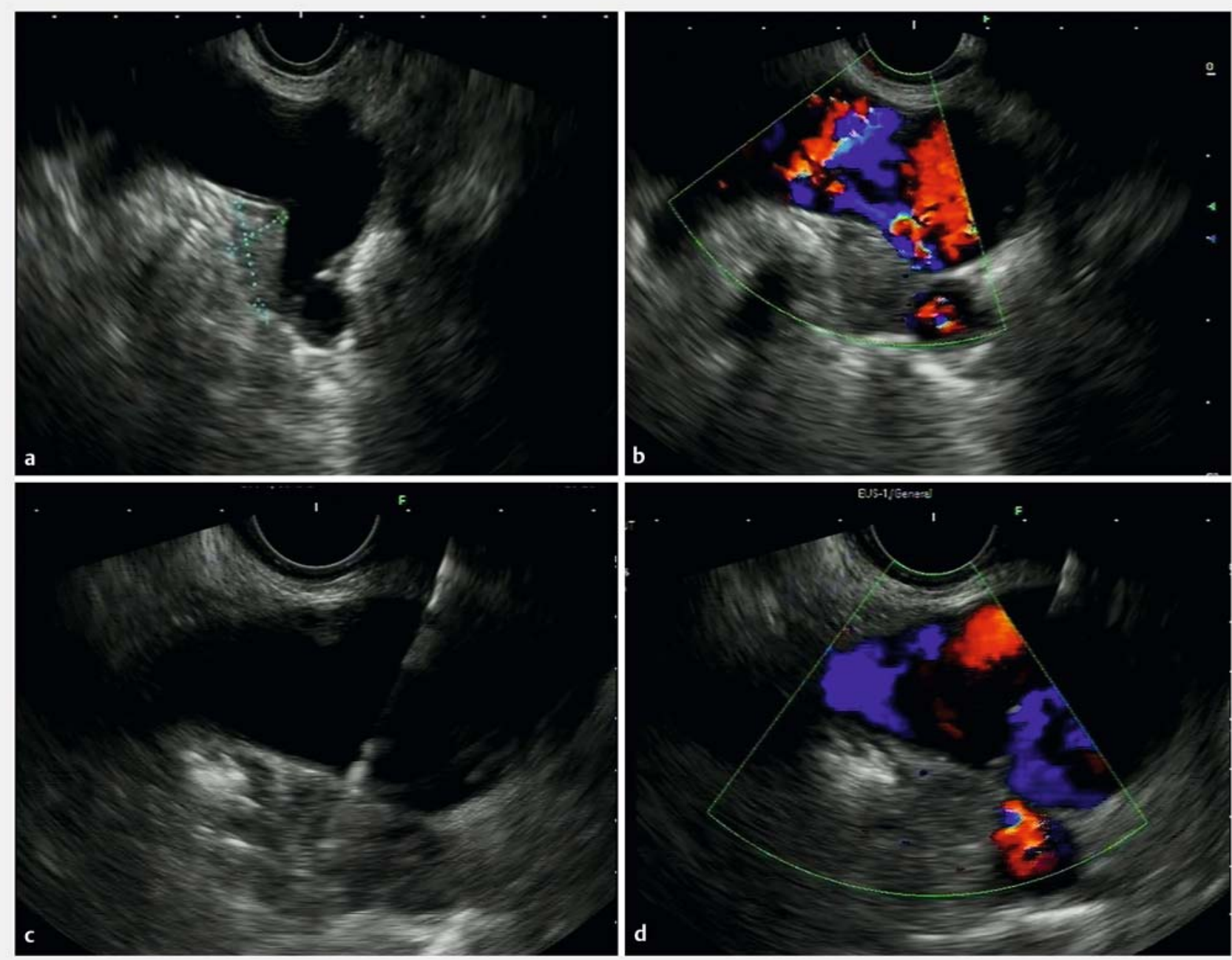

- Fig. 1 a, b A suspicious mediastinal lymph node located behind the left pulmonary artery, close to the aorto-pulmonary window. c A 25-G needle crossing the major vessel; the tip of the needle is seen in the target. $\mathbf{d}$ Doppler flow is detected in the pulmonary artery during the puncture.

\section{Introduction}

Sometimes a major vessel is interposed between the target lesion and the echoendoscope. Lesions of this kind are classically considered to be beyond the reach of the endosonographer.

Historically, the transvascular approach has been avoided because of concerns about bleeding, but the previous experience of radiologists has shown that traversing major vascular structures adjacent to the target biopsy site (i.e., aorta, cava, porta) does not increase the risk of adverse events (AEs) and should be considered when the result of the biopsy will affect the clinical strategy of patient care [1-3].

In recent years, a slow proliferation of studies regarding endoscopic ultrasound (EUS) and endobronchial ultrasound bronchoscopy (EBUS-guided transvascular biopsies, especially of intrathoracic lesions, has emerged.

EUS-guided transvascular fine-needle aspiration (EUS-TVFNA) has been described as a feasible and generally safe alternative approach in selected patients if other proven methods entail greater potential morbidity and no other target is available. This statement is based on small case series and retrospectives studies; to date, little is known about when EUS-TVFNA can be recommended [4].

The primary aim of this multicenter study was to analyze the diagnostic yield and safety of EUS-TV-FNA in thoracic and abdominal lesions. Secondary aims were to assess the clinical impact and technical aspects of the procedure.

\section{Patients and methods}

A retrospective multicenter study was designed, consisting of examination of a nationwide database involving all Spanish hospitals experienced in EUS-TV-FNA. All members of the Spanish Group of Endoscopic Ultrasound were invited by mail to participate (89 endoscopists). Six centers participated. The inclusion period ran from July 2007 to January 2020. Inclusion criteria were all EUS-TV-NA performed during the inclusion period. Ex- 

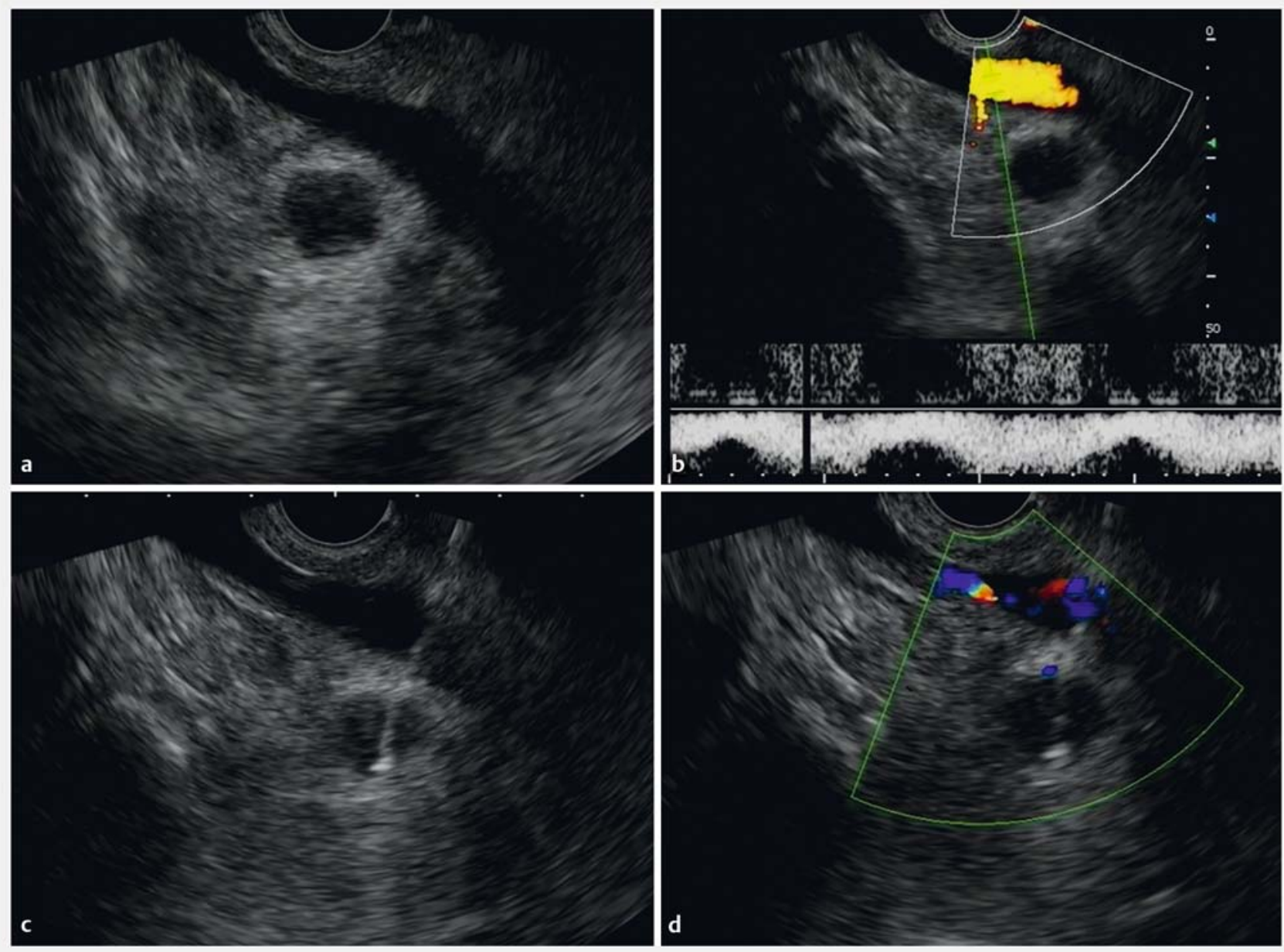

Fig. 2 a Abdominal lymph node suggestive of lymphoma with the inferior cava vein interposed. b Doppler effect in the cava vein. c, d A transcaval endoscopic-guided puncture with a $22-G$ needle is performed.

clusion criteria were coagulopathy, portal hypertension, and lack of follow-up information. The following variables were reviewed: demographic details, clinical data, staging imaging, procedure and technical details, cytological data, medication with potential risk of bleeding, follow-up data, incidents, and AEs. All imaging parameters were reviewed and taken from the original written reports.

\section{Technique}

All patients provided written informed consent before the procedure. All EUS-TV-FNA were performed by five experienced endosonographers (JCS, JRA, CDS, EVS, and JBG), each of whom had more than 15 years of experience in interventional EUS). Deep sedation was provided by a non-anesthesiologist or an anesthesiologist, depending on each center's protocol. For patients with antiplatelet or anticoagulant therapy, the recommendations of the international guidelines were followed [5]. Antibiotic prophylaxis was considered depending on each center's protocol.

Based on available imaging information, including the sameday EUS study, transvascular access was regarded as the only option to diagnose or stage these cases. The transvascular approach was considered only when the pathological information to be obtained would have an impact on the clinical plan, and it was subject to approval by a multidisciplinary committee.

A linear array echoendoscope (GF-UCT140-AL5, GF-UC160POL5 or GF-UCT180, Olympus; EG3870UTK, Pentax; or EG580UT, Fujifilm) was used to identify and puncture the target lesion. Prior to transvascular puncture, technical attempts were made to avoid an intravascular route (e. g., deflecting the tip or changing scope position). In the transaortic cases, a special effort was made to avoid intraluminal aortic plaques. Color Doppler imaging was used to avoid interposal vessels and to identify major vascular structures. The target was identified, and the needle was advanced, traversing the vessel until the tip was seen inside the lesion, and then the stylet was removed. The suction technique applied, if any (stylet slow-pull vs. standard suction), was applied, and needle type and size were selected at the discretion of the endosonographer (25 or 22G; EchoTip Ultra-HD, Cook or Expect Slimline, Boston Scientific). Fanning technique was avoided. The number of passes was deter- 
mined by the quantity of material or the presence of the cytopathology team.

Examples of different EUS-guided transvascular approaches are presented in $\mathbf{F i g . 1}$ and $\mathbf{F i g . 2}$, and Fig.S1).

At the end of the procedure, the needle was retracted, and the para-vascular area was observed for 2 minutes to assess potential immediate procedure-related AEs.

Samples were prepared with rapid on-site evaluation (ROSE) or without, depending on each center's protocol. All patients were monitored in the recovery room of the endoscopy unit for at least 6 hours, and at the discretion of each center were discharged or admitted for 24-hour clinical observation. No routine chest or abdominal imaging was done after the procedure.

AEs were defined and graded according to the ASGE lexicon severity grading system [6].

\section{Statistical analysis}

Categorical variables were described by the number of cases, percentages, and missing data. Continuous variables were described by the number of cases, mean and standard deviation, or the median and the interquartile range (IQR). Categorical variables were compared using the chi-squared test. Quantitative variables were compared using the student's $t$ test. Univariate analysis was performed to identify variables associated with incidents, cytopathological diagnoses and clinical impact. Multivariate analysis using multiple logistic regression was performed on predictor variables with a $P<0.05$.

Sensitivity, specificity, diagnostic accuracy, negative predictive value (NPV), and positive predictive value (PPV) were calculated. Positive biopsies were treated as true positive. Cases with non-representative material were considered as false negatives.

Diagnostic performance was considered as total amount of conclusive cytopathology results (malignancy plus benignity) excluding inconclusive results (atypical, suspected, or insufficient samples).

The level of statistical significance was set at $<0.05$. The statistical package used was SPSS version 22.0.

\section{Results}

\section{Demographics}

A total of 49 cases and 50 EUS-TV-FNA were collected, with a mean age of 64 years (SD 10.6); more than half were men (54\%). Demographic and clinical characteristics are shown in - Table 1.

Intrabdominal lesions were the most common targets ( $\mathrm{n}=$ $28)$, and the aorta $(n=19)$ and portal system $(n=17)$ the vessels most frequently punctured. Information related to types of vessels, target lesions, and their respective anatomical regions are summarized in > Table 2 .

\section{Interventional procedures}

In all 50 procedures, real-time visualization of the needle traversing the vessel and into the target was technically feasible and aspiration was applied in all. Most instances of deep seda-
- Table 1 Clinical and demographic characteristics.

Variables Data

$\mathrm{n}=49$ patients $/ 50$ procedures

\begin{tabular}{|l|l|}
\hline Age, mean (SD) & $64.4(10.6)$ \\
\hline Sex, $\mathrm{n}(\%)$ male/female & \\
\hline . Men & $27(54), 23(46.9)$ \\
\hline
\end{tabular}

$\mathrm{CCI}$, mean (SD)

No comorbidities, $\mathrm{n}(\%)$

$5.16(2.64)$

Cardiovascular risk factors, $\mathrm{n}$ (\%)

$9(18)$

Chronic pulmonary disease, $\mathrm{n}(\%)$

$32(64)$

Cardiovascular diseases, $\mathrm{n}(\%)$

$9(18)$

Chronic kidney disease, n (\%)

$12(24)$

Coagulopathy, n (\%)

4 (8)

Active neoplasia, $n$ (\%)

1 (2)

Antithrombotic drugs, n (\%)

- Antiplatelet

$12(24.4)$

- Oral anticoagulant

$0(0)$

ASA classification, $\mathrm{n}(\%)$

- 1

$5(10)$

- II

$22(44)$

- III

$23(46)$

- IV

$0(0)$

Sedative agents, $\mathrm{n}$ (\%)

- Propofol 39 (78)

- Fentanyl+midazolam

11 (22)

Sedation carried out by:

- Non-anesthesiologist $16(32)$

- Anesthesiologist 34 (68)

Inpatient treatment, $\mathrm{n}$ (\%)

- 6 hours observation in recovery room 29 (58)

- 24-hour admission to hospital $16(32)$

- Breakthrough admission $5(10.2)$

- Antibiotic prophylaxis, $\mathrm{n}(\%)$

ASA, American Society of Anesthesiologists; $\mathrm{CCl}$, Charlson Comorbidity Index; SD, standard deviation.

tion were controlled by anaesthesiologists (68\%), and the majority of patients were discharged after a period of observation of at least 6 hours (58\%). All centers except two contributed more than five cases. The median number of cases per center was 8.3 (range 1-18).

Endoscopist suspicion of malignancy, based on the EUS image, was the most common finding (70\%), and the most frequent final diagnoses were malignant mediastinal lymph nodes 
- Table 2 Vessels, target lesions, and respective anatomical regions.

\begin{tabular}{|c|c|c|c|c|c|c|c|}
\hline \multicolumn{2}{|l|}{ Vessels } & \multicolumn{6}{|c|}{ Target Locations } \\
\hline & & \multicolumn{2}{|l|}{ THORAX ${ }^{1}$} & \multicolumn{4}{|c|}{ ABDOMINAL ${ }^{2}$} \\
\hline & & Mediastinum & Lung & Pancreas & Non-pancreatic & Adrenal & Total \\
\hline \multirow{6}{*}{$\begin{array}{l}\text { - Arterial vessel } \\
n=29\end{array}$} & Aorta & 14 & 3 & $1^{3}$ & & & 18 \\
\hline & Pulmonary artery & 4 & & & & & 4 \\
\hline & Superior mesenteric artery & & & 2 & & & 2 \\
\hline & Hepatic artery & & & & 1 & & 1 \\
\hline & Splenic artery & & & 2 & 1 & & 3 \\
\hline & Gastroduodenal artery & & & 1 & & & 1 \\
\hline \multirow{5}{*}{$\begin{array}{l}\text { - Venous vessel } \\
n=20\end{array}$} & Porta & & & 9 & 3 & & 12 \\
\hline & Superior mesenteric vein & & & 1 & 4 & & 5 \\
\hline & Inferior vena cava & & & & 1 & 1 & 2 \\
\hline & Azygos & 1 & & & & & 1 \\
\hline & Splenic vein & & & 1 & & & 1 \\
\hline \multicolumn{2}{|l|}{ Total } & 19 & 3 & 17 & 10 & 1 & 50 \\
\hline
\end{tabular}

$(n=13)$, pancreatic tumours $(n=11)$ and lung cancer $(n=8)$. Mean (SD) of target diameter and distance to the target were $24 \mathrm{~mm}$ (14) and $21.3 \mathrm{~mm}$ (SD9.7) respectively. More data related to the EUS findings and final diagnoses are presented in - Table 3.

Technical aspects of FNA: needle size, 22 and $25 \mathrm{G}$ ( $52 \%$ and $48 \%$ respectively); aspiration technique, suction-syringe and slow-pull ( $44 \%$ and $58 \%$ respectively); median number of passes was two (interquartile range: 1.0 to 8.0). Other technical aspects of EUS-TV-FNA, including pathological and safety results, are summarized in $>$ Table 4 and Table S1. The diagnostic yield of EUS-TV-FNA was $86 \%$, representing malignant plus benign final results. Non-diagnostic samples were found in seven cases; these included dysplasia $(n=2)$, suspicious but not conclusive matter $(n=2)$, and insufficient matter $(n=3)$.

Intraluminal aorta plaques were found in three cases and changing the position of the scope was undertaken to avoid the plaques.

\section{Diagnostic performance}

A positive cytological diagnosis of malignancy was confirmed in 36 cases. Two suspected cytological diagnoses were confirmed as true positive with progression on follow-up imaging. Benign cytological results $(n=7)$ were confirmed by surgical biopsy $(n=1)$ and regression/stability on follow-up imaging procedures. Three insufficient samples were considered as false negative. The number of total false negatives rose to five, including two cases of pancreatic cystic tumors (dysplasia by cytological diagnosis). Only one considered false negative was confirmed as malignancy despite an insufficient sample with EUS (abdominal mass, diagnosed as lymphoma by percutaneous-guided biopsy in another more accessible point). The rest of false negatives were confirmed due to progression or suspicious behaviour in follow-up imaging tests The overall sensitivity, specificity, positive and negative predictive value, and accuracy were $88 \%(95 \% \mathrm{Cl}, 74-96), 100 \%(95 \% \mathrm{Cl}$, 59$100), 100 \%(95 \% \mathrm{Cl}, 88-100), 58 \%(95 \% \mathrm{Cl}, 38-76)$ and $90 \%$ (95\% Cl, 78-96) respectively (Table S2).

\section{Safety}

No AEs occurred, either during procedures or in the post-procedural period. Only three incidents were encountered. Two minor mural hematomas (aorta and pulmonary artery) were detected on EUS images immediately after a transvascular FNA. Self-limited bleeding after the EUS-guided puncture of a gastroduodenal artery was observed in a patient with antiplatelet therapy. None of these patients were symptomatic.

Twelve patients were treated with antiplatelet therapy without any additional cases of bleeding or hematomas. Antibiotic prophylaxis was administered in $38 \%$ of patients, and no cases of fever or infection related to the procedure were reported.

\section{Clinical impact}

Only in six cases did EUS-TV-FNA not cause a change in the global management of the patients. In most cases (88\%), the interventional procedure directly impacted the clinical management of the patient. 
- Table 3 Endoscopic ultrasound findings and diagnosis.

\begin{tabular}{|c|c|}
\hline Characteristics & Total, n-50 \\
\hline \multicolumn{2}{|l|}{ EUS findings: } \\
\hline - Diameter of the target, mean (SD), mm & $24.4(14.0)$ \\
\hline - Missing & $6(12.2)$ \\
\hline - Distance to the transducer, mean (SD), mm & $21.2(9.4)$ \\
\hline . Missing & $10(20.4)$ \\
\hline \multicolumn{2}{|l|}{ Echo pattern n (\%) } \\
\hline - Homogeneous & $20(40.8)$ \\
\hline - Heterogeneous & $29(59.2)$ \\
\hline \multicolumn{2}{|l|}{ Endoscopist suspicion, n (\%) } \\
\hline - Benignity & $6(12.2)$ \\
\hline - Malignancy & $34(69.4)$ \\
\hline " Undetermined & $9(18.4)$ \\
\hline \multicolumn{2}{|l|}{ Final diagnosis, $\mathrm{n}(\%)$ : } \\
\hline - Pancreatic adenocarcinoma & $13(26.5)$ \\
\hline - Metastases (LN and masses) & $11(22.4)$ \\
\hline - Lung cancer (LN and masses) & $8(16.3)$ \\
\hline - Mucinous pancreatic cyst & $4(8.2)$ \\
\hline - Benignity & $3(6.1)$ \\
\hline - Lymphoma & $2(4.1)$ \\
\hline - Others ${ }^{1}$ & $5(10.2)$ \\
\hline - Unrepresentative & $3(6.1)$ \\
\hline
\end{tabular}

\section{Univariate and multivariate analysis}

An arterial vessel and ASA III had a significant trend with incidents related to transvascular puncture. No significant factors were found to be related in clinical impact. Univariate analysis of incidents and final diagnoses is summarized in $>$ Table 4 and Table S3. On multivariate analysis, ROSE (OR6.2; $95 \% \mathrm{Cl}, 1.063-$ $36.737, P<0.043$ ) was found to be an independent predictor of obtaining a conclusive sample for a final diagnosis.

\section{Discussion}

Since Vincent et al and Wallace et al (2006 and 2007, respectively) published papers on the first transvascular (pulmonary artery and aorta) puncture guided by EBUS and EUS, respectively, only a few case reports and small case series have been published $[7,8]$. Much of the current literature on EUS-TV-FNA has paid special attention to intrathoracic lesions, and most of the knowledge about this technique comes from the EBUS field [912]. Many of the reports related to EUS-guided FNA involve the transaortic approach and focus on the study of lung cancer [4,
- Table 4 Univariate analysis of potential factors related to cytopathological diagnosis.

\begin{tabular}{|c|c|c|}
\hline Variables & Data & $P$ value $^{1}$ \\
\hline Age, mean (SD) & $64.4(10.6)$ & 0.05 \\
\hline Procedure time, median (IQR) & $30(24-75)$ & 0.08 \\
\hline FNA passes, median (IQR) & $2(1-8)$ & 0.51 \\
\hline \multicolumn{2}{|l|}{ Needle size, $\mathrm{n}(\%)$} & \multirow[t]{3}{*}{0.04} \\
\hline . 25-G & $26(52)$ & \\
\hline . 22-G & $25(51.0)$ & \\
\hline \multicolumn{2}{|l|}{$\mathrm{ROSE}^{2}, \mathrm{n}(\%)$} & \multirow[t]{3}{*}{0.02} \\
\hline - Yes & $33(66)$ & \\
\hline - No & $17(34.7)$ & \\
\hline \multicolumn{2}{|l|}{ FNA technique, n (\%) } & \multirow[t]{4}{*}{0.64} \\
\hline - Slow-pull (no syringe) & $27(54)$ & \\
\hline - Suction-syringe & $21(42.9)$ & \\
\hline - Others & $2(4.1)$ & \\
\hline \multicolumn{2}{|l|}{ EUS suspicious, $\mathrm{n}(\%)$} & \multirow[t]{4}{*}{0.17} \\
\hline - Benignity & $6(12.2)$ & \\
\hline - Malignancy & $35(70)$ & \\
\hline - Undetermined & $9(18.4)$ & \\
\hline \multicolumn{3}{|c|}{$\begin{array}{l}\text { FNA, fine needle aspiration; IQR, interquartile range; ROSE, rapid-on site } \\
\text { evaluation; SD, standard deviation. } \\
1 \text { P obtained with student's } t \text {-test and chi-squared test. Statistical signifi- } \\
\text { cance at } P<0.05 \\
{ }^{2} \text { Half of centers }(n-3) \text { had ROSE available. }\end{array}$} \\
\hline
\end{tabular}

8,13-15]. Other vascular interventions have included EUSguided portal access for diagnostic or therapeutic purposes in sampling for hepatocellular carcinoma and portal embolization [16-19].

To our knowledge, this is the first study of EUS-TV-FNA combining experience with intrathoracic and intrabdominal lesions and traversing different types of vessels with a considerable range of sample sizes. In the present study, a total of 50 transvasculars biopsies were taken, guided by EUS, and using 22-G and $25-G$ needles with an average of two passes. Intrabdominal lesions were more frequent targets than thoracic lesions, with the thoracic aorta and porta the most frequently traversed vessels.

An updated literature review is included ( $>$ Table 5 ), and three retrospective case series stand out for comparison to our results $[4,8,9,11-16,20,21]$.

Bartheld et al. performed 14 transaortic EUS-FNA in the diagnosis of mediastinal LN and lung tumors, using 25-G needles and a single pass. They reported a diagnostic yield of $71 \%$ and two hematomas (14\%) at the site of aortic puncture, without clinical consequences [4].

Wang et al. reported 26 transportal system EUS-guided transgastric procedures in diagnosing pancreatic tumours, using a 22-G needle, with an average of four passes, and a sen- 
- Table 5 Transaortic, transcarotid, transportal, and transcaval endoscopic ultrasound-guided fine-needle aspiration. A literature review.

\begin{tabular}{|c|c|c|c|c|c|c|c|c|}
\hline Author, y & Journal & $n$ & $\begin{array}{l}\text { Target } \\
\text { (n) }\end{array}$ & Vessel & $\begin{array}{l}\text { Needle } \\
\text { (size, model) }\end{array}$ & $\begin{array}{l}\text { Passes } \\
\text { median }\end{array}$ & $\begin{array}{l}\text { Diagnostic } \\
\text { yield }\end{array}$ & $\begin{array}{l}\text { AE or } \\
\text { incidents }\end{array}$ \\
\hline $\begin{array}{l}\text { Wallace MB } \\
2007\end{array}$ & $\begin{array}{l}\text { Ann Thor } \\
\text { Surg }\end{array}$ & 1 & LN & Thor. aorta & $\begin{array}{l}22-\mathrm{G} \\
\text { Cook }\end{array}$ & 1 & 1 & $\begin{array}{l}1 \text { small hema- } \\
\text { toma }\end{array}$ \\
\hline $\begin{array}{l}\text { Bartheld MB } \\
2009\end{array}$ & $\begin{array}{l}\text { Gastrointest } \\
\text { Endosc }\end{array}$ & 14 & $\begin{array}{l}\text { Lung (9) } \\
\operatorname{LN}(5)\end{array}$ & Thor. aorta & $\begin{array}{l}\text { 22-G } \\
\text { Hancke, Medi-Globe }\end{array}$ & 1 & $71 \%$ & $\begin{array}{l}2 \text { small hema- } \\
\text { tomas }\end{array}$ \\
\hline $\begin{array}{l}\text { Bang JY } \\
2012\end{array}$ & Am Surg & 1 & LN & Thor. aorta & $25-G$ & 1 & 1 & Noe \\
\hline $\begin{array}{l}\text { Lococo F } \\
2012\end{array}$ & Endoscopy & 1 & LN & Carotid & $\begin{array}{l}\text { 25-G } \\
\text { EchoTip, Cook }\end{array}$ & 3 & 1 & No \\
\hline $\begin{array}{l}\text { Lococo F } \\
2013\end{array}$ & Endoscopy & 1 & $\begin{array}{l}\text { Right } \\
\text { adrenal }\end{array}$ & Cava & $\begin{array}{l}\text { 22-G } \\
\text { EchoTip, Cook }\end{array}$ & 2 & 1 & No \\
\hline $\begin{array}{l}\text { Galasso } \\
2015\end{array}$ & Endoscopy & 1 & LN & Abd. aorta & $\begin{array}{l}25-G \\
\text { Echotip, } \\
\text { Cook }\end{array}$ & 3 & 1 & No \\
\hline $\begin{array}{l}\text { Lee NK } \\
2015\end{array}$ & $\begin{array}{l}\text { Gastrointest } \\
\text { Endosc }\end{array}$ & 1 & LN & Abd. aorta & $\begin{array}{l}25-G \\
\text { EchoTip, } \\
\text { Cook }\end{array}$ & 2 & 1 & No \\
\hline $\begin{array}{l}\text { Kazakov J } \\
2016\end{array}$ & $\begin{array}{l}\text { Ann Thor } \\
\text { Surg }\end{array}$ & 19 & LN & Thor Aorta & $22-G$ & - & $73 \%$ & No \\
\hline $\begin{array}{l}\text { Ravaglia C } \\
2019\end{array}$ & $\begin{array}{l}\text { Eur Clin } \\
\text { Respir J }\end{array}$ & 11 & LN & Thor aorta & $\begin{array}{l}\text { 21-G, 22-G } \\
\text { Olympus }\end{array}$ & 1 & $45 \%$ & No \\
\hline $\begin{array}{l}\text { Wang M } \\
2019\end{array}$ & $\begin{array}{l}\text { Ann Transl } \\
\text { Med }\end{array}$ & 26 & Pancreas & Porta, SMV & 22G, Cook & 4 & $92 \%$ & No \\
\hline $\begin{array}{l}\text { MolinaJC } \\
2020\end{array}$ & $\begin{array}{l}\text { J Thorac Car- } \\
\text { diovasc Surg }\end{array}$ & 65 & LN, Lung & Thor aorta & $22-G$ & 2 & $74 \%$ & $\begin{array}{l}1 \text { pseudoa- } \\
\text { neurysm }\end{array}$ \\
\hline $\begin{array}{l}\text { Present study } \\
2020\end{array}$ & - & 50 & $\begin{array}{l}\text { Thorax \& } \\
\text { abdominal }\end{array}$ & Multiples & $\begin{array}{l}\text { 22-G,25-G } \\
\text { Cook, Boston, } \\
\text { Olympus }\end{array}$ & 2 & $86 \%$ & $\begin{array}{l}3 \text { hematomas, } \\
1 \text { minor bleed- } \\
\text { ing }\end{array}$ \\
\hline
\end{tabular}

sitivity and accuracy of $91 \%$ and $92 \%$, respectively, without any AEs. The authors argued that the lower portal pressure compared with aortic pressure could explain why no hematomas were detected [16].

Recently, Molina et al. reported 65 cases of EUS-FNA, plus 35 EBUS cases of transvascular-guided biopsies of intrathoracic lesions, with the aorta the most common vessel traversed. The authors used a 22-G needle, with an average of two passes per target, and obtained an overall sensitivity and accuracy of $71.5 \%$ and $74.5 \%$, respectively. No immediate AEs were reported, and only one delayed aortic pseudoaneurysm. One possible explanation for this lower diagnostic performance is that the number of passes made in a transvascular approach is less than with standard EUS-FNA, and this might affect sample adequacy [12].

The diagnostic yield of our study was higher than in other studies. This may be because an average of two attempts (range: $1-8$ ) at transvascular FNA was made and ROSE was possible in most cases. Concretely, all transaortic cases, and all transportal cases except one, yielded a final diagnosis. On univariate analysis we found that a 25-G needle and ROSE were sig- nificantly related to obtaining a final diagnosis, but only ROSE was significant on the multivariate analysis. The fact that our endoscopists had extensive experience in the EUS-guided FNA technique surely contributed to these results.

In terms of safety, to date the reported AE rate in transvascular biopsies taken with EUS or EBUS guidance seems to be low and without fatal events. In the radiological literature, a rate of $0.05 \%$ major AEs related to translumbar aortography is reported, but asymptomatic mural hematomas are not uncommon [1-3]. To our knowledge, three immediate hematomas have been described after transaortic punctures guided by EUS, without the need for interventional therapy. One case of pseudo-aneurysm was detected 3 weeks after a transaortic EUS-FNA that was managed conservatively $[4,8,12]$.

In our study, no AEs were detected, and three incidents were reported: two hematomas after transaortic EUS-FNA using a $25-G$ needle ( 2 passes), and one self-limited bleed after a gastroduodenal puncture using a $22-\mathrm{G}$ needle (2 passes). No interventional procedures were required to manage these incidents. An arterial vessel and ASA III were almost significantly related to incidents, and no other factors were found to be related. Cur- 
iously, none of the transaortic cases with more than two passes was associated with incidents or AEs. Given these data, and their congruence with previously reported hematomas with transaortic approaches, we should offer a word of caution, and suggest that FNA should only be performed in the absence of alternative means to obtain a tissue diagnosis for para-aortic lesions.

Therefore, from a technical point of view, it seems reasonable to recommend, whenever possible, the use of 25-G needles and ROSE in these types of interventional procedures, especially in arteries, without limit to a single pass.

However, some questions remain: Does the use of thinner needle (25-G) really reduce the risk of bleeding? And does increasing the number of passes carry a greater risk of tumor seeding? To address these questions and their concerns, prospective studies are needed.

Finally, in addition to broad knowledge of echo-anatomy in the EUS field, a transvascular approach requires skills and expertise in the FNA technique. In our opinion, the degree of challenge depends on the vessel size. Crossing a splenic vessel and crossing the aorta with a needle are not the same. A transaortic or trans-pulmonary artery scenario must be recognized as being of greater complexity. The distance between the lesion and the tip of scope may be greater than $5 \mathrm{~cm}$ and predicting needle trajectory requires experience. The procedure should be performed by endoscopists with proven experience and with a favourable balance of benefits/risks.

\section{Limitations}

This study has some limitations, mainly owing to its retrospective design. First, variation between centers with a potential population bias cannot be excluded. Second, the variability of target lesions and different anatomical location of the vessels included in this study may have imposed a selection bias on the study population. Third, the lack of a standardized protocol with no specific follow-up implies a lack of information missing on follow-up, associated with a possible failure to catch some AEs.

\section{Conclusions}

This interventional technique is feasible and seems to be safe. The balance between diagnostic benefit and safety must be weighed. It is likely that a 25-G needle and ROSE may be recommended, especially in arteries; an endoscopic transvascular approach should be considered when no other locations are possible for taking samples in selected patients.

\section{Competing interest}

Drs. Gornals, Aparicio, and Vazquez-Sequeiros are consultants for Boston Scientific.

\section{References}

[1] Lieberman RP, Waldman SD. Celiac plexus neurolysis with the modified transaortic approach. Radiology 1990; 175: 274-276

[2] Gupta S, Ahrar K, Morello F et al. Masses around the pancreatic head: CT-guided coaxial fine-needle aspiration biopsy with a posterior transcaval approach. Radiology 2002; 222: 63-69

[3] Sofocleous CT, Schubert ], Brown KT et al. CT-guided transvenous or transcaval needle biopsy of pancreatic and peripancreatic lesions. J Vasc Intern radiol 2004; 15: 1099-1104

[4] von Bartheld MB, Rabe KF, Annema JT. Transaortic EUS-guided FNA in the diagnosis of lung tumors and lymph nodes. Gastrointest Endosc 2009; 69: 345-349

[5] Veitch AM, Vanbiervliet G, Gershlick AH et al. Endoscopy in patients on antiplatelet or anticoagulant therapy, including direct oral anticoagulants: British Society of Gastroenterology (BSG) and European Society of Gastrointestinal Endoscopy (ESGE) guidelines. Endoscopy 2016; 48: 385-402

[6] Cotton PB, Eisen GM, Aabakken L et al. A lexicon for endoscopic adverse events: report of an ASGE workshop. Gastrointest Endosc 2010; 71: 446-454

[7] Vincent B, Huggins JT, Doelken P et al. Successful real-time endobronchial ultrasound-guided transbronchial needle aspiration of a hilar lung mass obtained by traversing the pulmonary artery. J Thorac Oncol 2006; 1: 362-364

[8] Wallace MB, Woodward TA, Raimondo M et al. Transaortic fine-needle aspiration of centrally located lung cancer under endoscopic ultrasound guidance: The Final Frontier. Ann Thorac Surg 2007; 84: 10191021

[9] Kazakov ], Hegde P, Tahiri M et al. Endobronchial and endoscopic ultrasound-guided transvascular biopsy of mediastinal, hilar, and lung lesions. Ann Thoracic Surg 2017; 103: 951-955

[10] Mehta RM, Biraris PR, Pattabhiraman V et al. Defining expanded areas in EBUS sampling: EBUS guided trans- and intra-pulmonary artery needle aspiration, with review of transvascular EBUS. Clin Respir J 2018; 12: 1958-1963

[11] Ravaglia C, Colella S, Tomassetti S et al. Diagnostic yield and safety of EUS-FNA biopsy in sub-aortic and para-aortic lymph node stations with the trans-aortic approach: a case series and literature review. Eur Clin Respir J; 2019: Available from (Accessed 2020 March 24): 6: 1552064. https://www.tandfonline.com/doi/full/10.1080/ 20018525.2018.1552064?af=R

[12] Molina JC, Chaudry F, Menezes V et al. Transvascular Endosonographic-Guided Needle Biopsy of Intrathoracic Lesions. J Thorac Cardiovasc Surg 2019: doi:10.1016/j.jtcvs.2019.10.017

[13] Bang JY, Varadarajulu S. Diagnosis of a metastatic neuroendocrine tumor of the pancreas by transaortic endoscopic ultrasound-guided fine-needle aspiration. Am Surg 2012; 78: 183-184

[14] Galasso D, Rimba M, Attili F et al. Transaortic endoscopic ultrasoundguided fine-needle aspiration of a positron emission tomographypositive abdominal para-aortic lymph node. Endoscopy 2015; 47: 536-537

[15] Lee NK, Park S, Park S et al. EUS-guided trans-aortic fine-needle aspiration for intra-abdominal plasmacytoma in a patient with multiple myeloma. Gastrointest Endosc 2015; 81: 1479-1480

[16] Wang M, Huang S, Pei R et al. Endoscopic ultrasonography guided transgastric trans-portal system fine needle aspiration for diagnosing pancreatic head and uncinate process malignancy. Ann Transl Med 2019; 7: 719

[17] Garg R, Rustagi T. Endoscopic ultrasound-guided portal venous access: diagnostic and therapeutic implications. J Clin Gastroenterol 2017; 51: 677-682 
[18] Trikudanathan G, Pannala R. ASGE Technology Committee. et al. EUSguided portal vein interventions. Gastrointest Endosc 2017; 85: 883888

[19] Chapman CG, Waxman I. EUS-guided portal vein sampling. EndosC Ultrasound 2018; 7: 240-245
[20] Lococo F, De Vincentis F, Naldini A et al. Transesophageal endoscopic ultrasound-guided transcarotid fine needle aspiration of a positron emission tomography (PET)-positive mediastinal lymph node. Endoscopy 2012; 44: 402-403

[21] Lococo F, Attili F, Meacci E et al. Transcaval endoscopic ultrasoundguided fine needle aspiration of a right adrenal lesion. Endoscopy 2013; 45: 201-202 